\title{
Fixed-Time Flocking Problem of a Cucker-Smale Model
}

\author{
Fen Nie and Yicheng Liu $(\mathbb{D}$ \\ College of Liberal Arts and Sciences, National University of Defense Technology, Changsha 410073, China \\ Correspondence should be addressed to Yicheng Liu; liuyc2001@hotmail.com
}

Received 1 January 2020; Revised 22 April 2020; Accepted 12 May 2020; Published 31 May 2020

Academic Editor: Nazrul Islam

Copyright (c) 2020 Fen Nie and Yicheng Liu. This is an open access article distributed under the Creative Commons Attribution License, which permits unrestricted use, distribution, and reproduction in any medium, provided the original work is properly cited.

\begin{abstract}
In this paper, we studied a Cucker-Smale model with continuous non-Lipschitz protocol. The methodology presented in the current work is based on the explicit construction of a Lyapunov functional. By using the fixed-time control technology, we show that the flocking can occur in fixed time if the communication rate function is locally Lipschitz continuous and has a lower bound, and we can obtain the estimation of the converging time which is independent of the initial states of agents. Theoretical results are supported by numerical simulations.
\end{abstract}

\section{Introduction}

Collective motions refers to an orderly movement organized by agents with limited environmental information and simple rules. In recent years, the study on collective motions has gained increasing interest in robotics, control theory, economics, and social sciences $[1,2]$. Several mathematical models have been proposed [3-6] to characterize the mechanism of collective flocking motion without central direction. Among others, the celebrated Cucker-Smale model [5] provided a framework to explain the self-organizing behavior in various complex systems, and the model is given by the following ODE system:

$$
\left\{\begin{array}{l}
\frac{\mathrm{d} x_{i}}{\mathrm{~d} t}=v_{i}, t>0, \quad i=1,2, \cdots, N, \\
\frac{\mathrm{d} v_{i}}{\mathrm{~d} t}=k \sum_{j=1}^{N} a_{i j}\left(\left\|x_{j}-x_{i}\right\|\right)\left(v_{j}-v_{i}\right),
\end{array}\right.
$$

subject to the initial configuration $\left(x_{i}(0), v_{i}(0)\right)=\left(x_{i 0}, v_{i 0}\right)$, where $N$ denotes the number of particles, $k$ measures the interaction strength, $x_{i}$ and $v_{i}$ denote the position and velocity of the $i$ th particle at the time $t$, and $a_{i j}$ measures the influence intensity quantified by the pairwise influence of particle $j$ on the alignment of particle $i$. It is a function of the distance of two particles defined as

$$
a_{i j}^{\mathrm{CS}}\left(\left\|x_{j}-x_{i}\right\|\right)=\frac{\psi\left(\left\|x_{j}-x_{i}\right\|\right)}{N},
$$

where $\psi(r)=\left(1 /\left(1+r^{2}\right)^{\beta}\right)$ is called the influence function, $\beta \geq 0$. In [5], the authors showed that the unconditional flocking occurs when $\beta<(1 / 2)$, while the conditional flocking occurs under some restricted conditions on the initial data setting when $\beta \geq(1 / 2)$. In [7], the authors extended the conclusions of unconditional flocking to $\beta \leq(1 / 2)$ by the energy method. In [8], the authors introduced a nonsymmetric influence function and took into account relative distance between agents instead of the distance between agents:

$$
a_{i j}^{\mathrm{MT}}\left(\left\|x_{j}-x_{i}\right\|\right)=\frac{\psi\left(\left\|x_{j}-x_{i}\right\|\right)}{\sum_{1 \leq k \leq N} \psi\left(\left\|x_{k}-x_{i}\right\|\right)} .
$$

Based on the notion of active sets, a sufficient condition for flocking was derived. Recently, there are many extensive observations and improvements to the Cucker-Smale model. See, for examples, time delay is introduced in [9-12], collision avoidance is considered in [13-16], and hierarchical structure is involved in [12, 17-20]. However, the flocking phenomenon described in the most previous works is an asymptotic behaviour, which means that the flocking can only occur when time approaches to infinity. Then, a natural question is that whether the system undergoes flocking 
behaviours within a finite time? In fact, under some occasional perturbations, individuals in bird flocks or fish schools can return back to ordered group motion after adjusting their states in a short time. Recently, there are few contributions to the Cucker-Smale model by using finitetime control theory. In [21], when the communication rate function is locally Lipschitz continuous and has a lower bound, the authors obtain the finite-time flocking by constructing a Lyapunov functional. In [22], the authors modified the Cucker-Smale model with continuous nonLipschitz protocol. When the influence function has a singular interval, the system will undergo a flocking evolution in finite time, and the minimum distance between agents in the flocking evolution process is greater than the control parameter. Although finite-time flocking performance has favourable properties, the estimation of convergence time usually depends on initial states of networked particles. It will restrict the applications in practice if the initial conditions are unavailable previously. In the similar performance, there are lots of works in the field of fix-time consensus [23-26]. However, there is little work about the fixed-time flocking performance of the Cucker-Smale model.

The main purpose of this article is to investigate the fixed-time flocking performance of a Cucker-Smale model. The remaining of this paper is organized as follows. In Section 2, a Cucker-Smale model with continuous nonLipschitz protocol is presented, and some useful preliminaries are also given in this section. In Section 3, the sufficient conditions for fixed-time flocking are established, and the numerical simulations are provided to validate the theoretical results in Section 4. Finally, the conclusions are drawn in Section 5.

\section{Problem Statement and Preliminaries}

In this section, we consider an $\mathrm{N}$-agent model with nonlinear terms. Let $x_{i}=\left(x_{i}^{1}, x_{i}^{2}, \ldots, x_{i}^{d}\right) \in \mathbb{R}^{d}$, $v_{i}=\left(v_{i}^{1}, v_{i}^{2}, \ldots, v_{i}^{d}\right) \in \mathbb{R}^{d}$ denote the position and velocity of the $i$ th agent at the time $t$, respectively. The modified Cucker-Smale model in this paper can be described by the following equations:

$$
\left\{\begin{array}{l}
\frac{\mathrm{d} x_{i}}{\mathrm{~d} t}=v_{i}, t>0, \quad i=1,2, \cdots, N, \\
\frac{\mathrm{d} v_{i}}{\mathrm{~d} t}=\frac{k_{1}}{N} \sum_{j=1}^{N} \psi\left(\left\|x_{j}-x_{i}\right\|\right) \operatorname{sig}\left(v_{j}-v_{i}\right)^{p}+\frac{k_{2}}{N} \sum_{j=1}^{N} \psi\left(\left\|x_{j}-x_{i}\right\|\right) \operatorname{sig}\left(v_{j}-v_{i}\right)^{q},
\end{array}\right.
$$

subject to initial configuration

$$
\left(x_{i}(0), v_{i}(0)\right)=\left(x_{i 0}, v_{i 0}\right),
$$

where $p$ and $q$ are two constants with $0<p<1<q . k_{1}$ and $k_{2}$ measure the interaction strengths, $\psi$ is defined in (2), and

$$
\operatorname{sig}\left(v_{j}-v_{i}\right)^{p}=\left\{\operatorname{sgn}\left(v_{j}^{1}-v_{i}^{1}\right)\left|v_{j}^{1}-v_{i}^{1}\right|^{p}, \ldots, \operatorname{sgn}\left(v_{j}^{d}-v_{i}^{d}\right)\left|v_{j}^{d}-v_{i}^{d}\right|^{p}\right\},
$$

where $\operatorname{sgn}(\cdot)$ is the signum function:

$$
\operatorname{sgn}(s)= \begin{cases}1, & s>0, \\ 0, & s=0, \\ -1, & s<0 .\end{cases}
$$

At this stage, we list the following lemmas, which play an important role in the proof of the main results.

Lemma 1 (special case with $k=1$ in [27]). Consider the following equation:

$$
\begin{aligned}
\dot{x} & =f(t, x), \\
x(0) & =x_{0},
\end{aligned}
$$

where $x \in \mathbb{R}^{n}$ and $f: \mathbb{R}_{+} \times \mathbb{R}^{n} \longrightarrow \mathbb{R}^{n}$ is a nonlinear continuous function. Assume the origin is an equilibrium point of
(8). If there exists a continuous radially unbounded function $H: \mathbb{R}^{n} \longrightarrow \mathbb{R}_{+} \cup\{0\}$ such that

(i) $H(z)=0 \Leftrightarrow z=0$.

(ii) If there are some positive constants $\vartheta, \delta, a$, and $b$ such that $0<a<1<b$ and the inequality

$$
\dot{H}(z(t)) \leq-\vartheta H^{a}(z(t))-\delta H^{b}(z(t)),
$$

holds for any solution $z(t)$ of (8), then the origin is globally fixed-time stable, and $H(t) \equiv 0$ if

$$
t \geq \frac{1}{9(1-a)}+\frac{1}{\delta(b-1)} .
$$

Lemma 2 (see [28]). Let $y \in \mathbb{R}^{n}$ and $0<r<s$; then, the following inequalities hold:

$$
\begin{gathered}
\left(\sum_{i=1}^{n}\left|y_{i}\right|^{s}\right)^{1 / s} \leq\left(\sum_{i=1}^{n}\left|y_{i}\right|^{r}\right)^{1 / r}, \\
\left(\frac{1}{n} \sum_{i=1}^{n}\left|y_{i}\right|^{s}\right)^{1 / s} \geq\left(\frac{1}{n} \sum_{i=1}^{n}\left|y_{i}\right|^{r}\right)^{1 / r} .
\end{gathered}
$$




\section{Sufficient Conditions for Fixed- Time Flocking}

In this section, we shall show that systems (4) and (5) with continuous non-Lipschitz protocol have fixed-time flocking. First, we first introduce the definition of the fixed-time flocking.

Definition 1. Systems (4) and (5) are said to reach fixed-time flocking if and only if the systems satisfy the following two conditions:

(i) Velocity alignment: the velocity fluctuations go to zero in the fixed-time $T$; the time function $T$ is called the convergence time independent of the initial values.

$$
\left\|v_{i}-v_{j}\right\|=0, \quad \forall t \geq T \text { for } i, j=1,2, \cdots, N
$$

(ii) Forming a group: the position fluctuations are uniformly bounded in time $t$ :

$$
\sup _{0 \leq t \leq \infty}\left\|x_{i}-x_{j}\right\|<\infty, \quad \text { for } i, j=1,2, \cdots, N
$$

Theorem 1. Consider the Cucker-Smale model ((4) and (5)) and assume that the communication rate function $\psi$ is locally Lipschitz continuous with a lower bound, that is, there exists $\psi^{*}>0$ such that $\inf _{s>0} \psi(s) \geq \psi^{*}$. Then, systems (4) and (5) reach fixed-time flocking. And the convergence time is given by

$$
T \leq T^{*} \doteq \frac{2}{\vartheta(1-p)}+\frac{2}{\delta(q-1)},
$$

where $\vartheta=k_{1} \psi^{*} 2^{(p+1) / 2} N^{(p-1) / 2}$ and $\delta=k_{2} \psi^{*} 2^{(q+1) / 2} d^{(1-q) / 2}$.

Proof. Firstly, we consider macroscopic variables:

$$
x_{c}=\frac{1}{N} \sum_{i=1}^{N} x_{i}, v_{c}=\frac{1}{N} \sum_{i=1}^{N} v_{i} \text {. }
$$

By the symmetry of the indices, we have

$$
\sum_{i=1}^{N} \frac{\mathrm{d} v_{i}}{\mathrm{~d} t}=\frac{k_{1}}{N} \sum_{i=1}^{N} \sum_{j=1}^{N} \psi\left(\left\|x_{j}-x_{i}\right\|\right) \operatorname{sig}\left(v_{j}-v_{i}\right)^{p}+\frac{k_{2}}{N} \sum_{i=1}^{N} \sum_{j=1}^{N} \psi\left(\left\|x_{j}-x_{i}\right\|\right) \operatorname{sig}\left(v_{j}-v_{i}\right)^{q}=0
$$

Hence, the explicit dynamics for the macroscopic variables is given as

$$
\begin{aligned}
\frac{\mathrm{d} x_{c}}{\mathrm{~d} t} & =v_{c}, \\
\frac{\mathrm{d} v_{c}}{\mathrm{~d} t} & =0,
\end{aligned}
$$

which implies that

$$
\begin{aligned}
v_{c}(t) & =v(0), \\
x_{c}(t) & =x_{c}(0)+t v_{c}(0), \\
t & \geq 0 .
\end{aligned}
$$

Introducing the fluctuations $\left(\widehat{x}_{i}, \widehat{v}_{i}\right)$,

$$
\begin{aligned}
& \widehat{x}_{i} \doteq x_{i}-x_{c}, \\
& \widehat{v}_{i} \doteq v_{i}-v_{c} .
\end{aligned}
$$

Then, systems (4) and (5) can be written as

$$
\left\{\begin{array}{l}
\frac{\mathrm{d} \widehat{x}_{i}}{\mathrm{~d} t}=\widehat{v}_{i}, t>0, \quad i=1,2, \cdots, N, \\
\frac{\mathrm{d} \widehat{v}_{i}}{\mathrm{~d} t}=\frac{k_{1}}{N} \sum_{j=1}^{N} \psi\left(\left\|\widehat{x}_{j}-\widehat{x}_{i}\right\|\right) \operatorname{sig}\left(\widehat{v}_{j}-\widehat{v}_{i}\right)^{p}+\frac{k_{2}}{N} \sum_{j=1}^{N} \psi\left(\left\|\widehat{x}_{j}-\widehat{x}_{i}\right\|\right) \operatorname{sig}\left(\widehat{v}_{j}-\widehat{v}_{i}\right)^{q},
\end{array}\right.
$$


with the initial value

$$
\left(\widehat{x}_{i}(0), \widehat{v}_{i}(0)\right)=\left(\widehat{x}_{i 0}, \widehat{v}_{i 0}\right) .
$$

For convenience, we remove the hat out of the variables and also use $\left(x_{i}, v_{i}\right)$ instead of $\left(\widehat{x}_{i}, \widehat{v}_{i}\right)$. It is easy to see that

$$
\sum_{i=1}^{N} x_{i}=0, \sum_{i=1}^{N} v_{i}=0
$$

Take the candidate Lyapunov function

$$
V(t) \doteq \sum_{i=1}^{N}\left\|v_{i}\right\|^{2}, X(t) \doteq \sum_{i=1}^{N}\left\|x_{i}\right\|^{2} .
$$

Then, we have

$\sum_{1 \leq i, j \leq N}\left\|v_{j}-v_{i}\right\|^{2}=2 N \sum_{i=1}^{N}\left\|v_{i}\right\|^{2}-2\left\langle\sum_{i=1}^{N} v_{i}, \sum_{j=1}^{N} v_{j}\right\rangle=2 N V$,

$\sum_{1 \leq i, j \leq N}\left\|x_{j}-x_{i}\right\|^{2}=2 N \sum_{i=1}^{N}\left\|x_{i}\right\|^{2}-2\left\langle\sum_{i=1}^{N} x_{i}, \sum_{j=1}^{N} x_{j}\right\rangle=2 N X$.

It is easy to see that the velocity difference of all individuals will tend to zero in fixed time if the function $V(t)$ tends to 0 in fixed time. And the diameter of a group is bounded if the function $X(t)$ is bound.

From Lemma 2, using the fact $\inf _{s>0} \psi(s) \geq \psi^{*}$, we see that

$$
\begin{aligned}
\left|\frac{\mathrm{d} V}{\mathrm{~d} t}\right|= & \left|\frac{\mathrm{d}}{\mathrm{d} t} \sum_{i=1}^{N}\left\|v_{i}\right\|^{2}\right|=\left|2 \sum_{i=1}^{N}\left\langle v_{i}, \dot{v}_{i}\right\rangle\right| \\
= & \frac{2 k_{1}}{N}\left\langle\sum_{1 \leq i, j \leq N} \psi\left(\left\|x_{j}-x_{i}\right\|\right) \operatorname{sig}\left(v_{j}-v_{i}\right)^{p}, v_{i}\right\rangle \\
& +\frac{2 k_{2}}{N}\left\langle\sum_{1 \leq i, j \leq N} \psi\left(\left\|x_{j}-x_{i}\right\|\right) \operatorname{sig}\left(v_{j}-v_{i}\right)^{q}, v_{i}\right\rangle \\
= & -\frac{k_{1}}{N}\left\langle\sum_{1 \leq i, j \leq N} \psi\left(\left\|x_{j}-x_{i}\right\|\right) \operatorname{sig}\left(v_{j}-v_{i}\right)^{p},\left(v_{j}-v_{i}\right)\right\rangle \\
& -\frac{k_{2}}{N}\left\langle\sum_{1 \leq i, j \leq N} \psi\left(\left\|x_{j}-x_{i}\right\|\right) \operatorname{sig}\left(v_{j}-v_{i}\right)^{q},\left(v_{j}-v_{i}\right)\right\rangle \\
\leq & -\frac{k_{1}}{N} \psi^{*} \sum_{1 \leq i, j \leq N} \sum_{k=1}^{d}\left|v_{j k}-v_{i k}\right|^{p+1}-\frac{k_{2}}{N} \psi^{*} \sum_{1 \leq i, j \leq N} \sum_{k=1}^{d}\left|v_{j k}-v_{i k}\right|^{q+1} .
\end{aligned}
$$

Note that $0<p<1<q$. Then, employing Lemma 2, one can easily obtain

$$
\begin{aligned}
& \left(\sum_{k=1}^{d}\left|v_{j k}-v_{i k}\right|^{p+1}\right)^{1 /(p+1)} \geq\left(\sum_{k=1}^{d}\left|v_{j k}-v_{i k}\right|^{2}\right)^{1 / 2}=\left\|v_{j}(t)-v_{i}(t)\right\|, \\
& \left(\frac{1}{d} \sum_{k=1}^{d}\left|v_{j k}-v_{i k}\right|^{q+1}\right)^{1 /(q+1)} \geq\left(\frac{1}{d} \sum_{k=1}^{d}\left|v_{j k}-v_{i k}\right|^{2}\right)^{1 / 2} .
\end{aligned}
$$

Thus, we have

$$
\begin{aligned}
& \sum_{k=1}^{d}\left|v_{j k}-v_{i k}\right|^{p+1} \geq\left\|v_{j}(t)-v_{i}(t)\right\|^{p+1} \\
& \sum_{k=1}^{d}\left|v_{j k}-v_{i k}\right|^{q+1} \geq d\left(\frac{1}{d} \sum_{k=1}^{d}\left|v_{j k}-v_{i k}\right|^{2}\right)^{(q+1) / 2}=d^{(1-q) / 2}\left\|v_{j}(t)-v_{i}(t)\right\|^{q+1} .
\end{aligned}
$$

Let $s=1$ and $r=((p+1) / 2)$; by applying (11) and (24) to the processing inequality, we show that

$$
\sum_{i, j=1}^{N}\left(\left\|v_{j}(t)-v_{i}(t)\right\|^{2}\right)^{(p+1) / 2} \geq\left(\sum_{i, j=1}^{N}\left\|v_{j}(t)-v_{i}(t)\right\|^{2}\right)^{(p+1) / 2}=(2 N V)^{(p+1) / 2} .
$$


Similarly, let $s=((q+1) / 2)$ and $r=1$; by applying (11) and (24), we get that

$$
\sum_{i, j=1}^{N}\left(\left\|v_{j}(t)-v_{i}(t)\right\|^{2}\right)^{(q+1) / 2} \geq N^{(1-q) / 2}\left(\sum_{i, j=1}^{N}\left\|v_{j}(t)-v_{i}(t)\right\|^{2}\right)^{(q+1) / 2}=N^{(1-q) / 2}(2 N V)^{(q+1) / 2}
$$

Hence, we conclude

$\frac{\mathrm{d} V}{\mathrm{~d} t} \leq-k_{1} \psi^{*} 2^{(p+1) / 2} N^{(p-1) / 2} V^{(p+1) / 2}-k_{2} \psi^{*} 2^{(q+1) / 2} d^{(1-q) / 2} V^{(q+1) / 2}$.

Finally, from Lemma 1 , when $a=((p+1) / 2)$ and $b=((q+1) / 2)$, we have

$$
V(t) \equiv 0, \quad t \geq T
$$

and the convergence time independent of the initial values is estimated by

$$
\begin{aligned}
T \leq T^{*} \doteq & \frac{1}{\vartheta(1-((p+1) / 2))}+\frac{1}{\delta(((q+1) / 2)-1)} \\
& =\frac{2}{\vartheta(1-p)}+\frac{2}{\delta(q-1)},
\end{aligned}
$$

where $\vartheta=k_{1} \psi^{*} 2^{(p+1) / 2} N^{(p-1) / 2}$ and $\delta=k_{2} \psi^{*} 2^{(q+1) / 2} d^{(1-q) / 2}$. Thus, from (24), we achieve

$$
v_{i}(t) \equiv 0, \quad \forall t \geq T, i=1,2, \cdots, N .
$$

This implies that condition (i) of the definition of fixedtime flocking holds.

Now, we prove condition (ii) of the definition of fixedtime flocking is also true. It is necessary to show that the function $X(t)$ is bounded.

It follows from (31) that $V(t)$ is a nonincreasing function with respect to $t$. That is, when $t>0, V(0) \geq V(t) \geq 0$. By using the triangle inequality and Cauchy-Schwarz inequality, we have

$$
\frac{\mathrm{d} X}{\mathrm{~d} t}=2 \sum_{i=1}^{N}\left\langle x_{i}, v_{i}\right\rangle \leq 2 \sum_{i=1}^{N}\left\|x_{i}\right\|\left\|v_{i}\right\| \leq 2 X^{1 / 2} V^{1 / 2} .
$$

Integrating the differential inequality (35) from 0 to $t$ yields that

$$
X^{1 / 2}(t) \leq X^{1 / 2}(0)+\int_{0}^{t} V^{1 / 2}(s) \mathrm{d} s .
$$

If $t<T$, then it is deduced from (36) that

$X^{1 / 2}(t) \leq X^{1 / 2}(0)+\int_{0}^{T} V^{1 / 2}(t) \mathrm{d} t \leq X^{1 / 2}(0)+V^{1 / 2}(0) T<\infty$.

If $t>T$, then it is deduced from (31) and (36) that

$$
\begin{aligned}
X^{1 / 2}(t) & \leq X^{1 / 2}(0)+\int_{0}^{T} V^{1 / 2}(t) \mathrm{d} t+\int_{T}^{t} V^{1 / 2}(t) \mathrm{d} t \\
& \leq X^{1 / 2}(0)+\int_{0}^{T} V^{1 / 2}(t) \mathrm{d} t \\
& \leq X^{1 / 2}(0)+V^{1 / 2}(0) T<\infty .
\end{aligned}
$$

Thus,

$$
\sup _{0 \leq t \leq \infty}\left\|x_{i}-x_{j}\right\|^{2}<\infty, \quad \text { for } i, j=1,2, \cdots, N .
$$

This completes the proof.

Remark 1. Compared to [21], we added the term

$$
\frac{k_{2}}{N} \sum_{j=1}^{N} \psi\left(\left\|x_{j}-x_{i}\right\|\right) \operatorname{sig}\left(v_{j}-v_{i}\right)^{q} \text {, }
$$

to the control protocol; the advantage of Theorem 1 is that the convergence time is independent of the initial states of agents which is estimated by

$$
\begin{aligned}
T \leq T^{*} \doteq & \frac{2}{k_{1} \psi^{*} 2^{(p+1) / 2} N^{(p-1) / 2}(1-p)} \\
& +\frac{2}{k_{2} \psi^{*} 2^{(q+1) / 2} d^{(1-q) / 2}(q-1)}
\end{aligned}
$$

However, in [21], the convergence time is estimated by

$$
T \leq T^{*} \doteq \frac{2\left(\sum_{i=1}^{N}\left\|v_{i}(0)\right\|^{2}\right)^{(1-p) / 2} N^{-((1+p) / 2)}}{\psi^{*} 2^{(1+p) / 2}(1-p)},
$$

which is formulated by the initial speed of all agents.

\section{Simulations}

In this section, we choose some special initial values and parameters to verify our results. Let $N=30, p=0.2, q=2$, $k_{1}=1, k_{2}=2, d=2$, Using Euler algorithm, step length $h=0.01$, and

$$
\psi(r)= \begin{cases}\frac{1}{\left(1+r^{2}\right)^{\beta}}, & \text { if } r \leq r^{*}, \\ \frac{1}{\left(1+\left(r^{*}\right)^{2}\right)^{\beta}}, & \text { if } r>r^{*},\end{cases}
$$




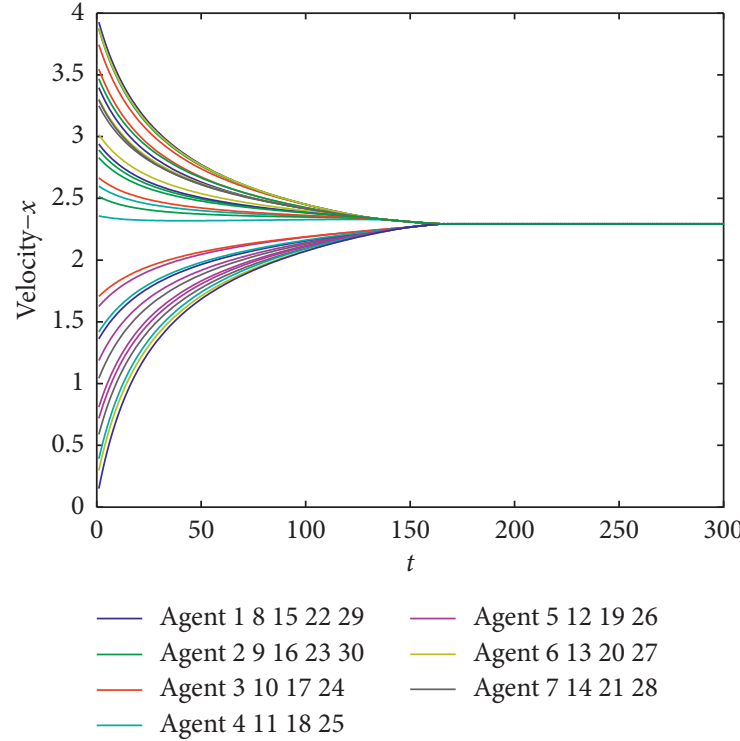

(a)

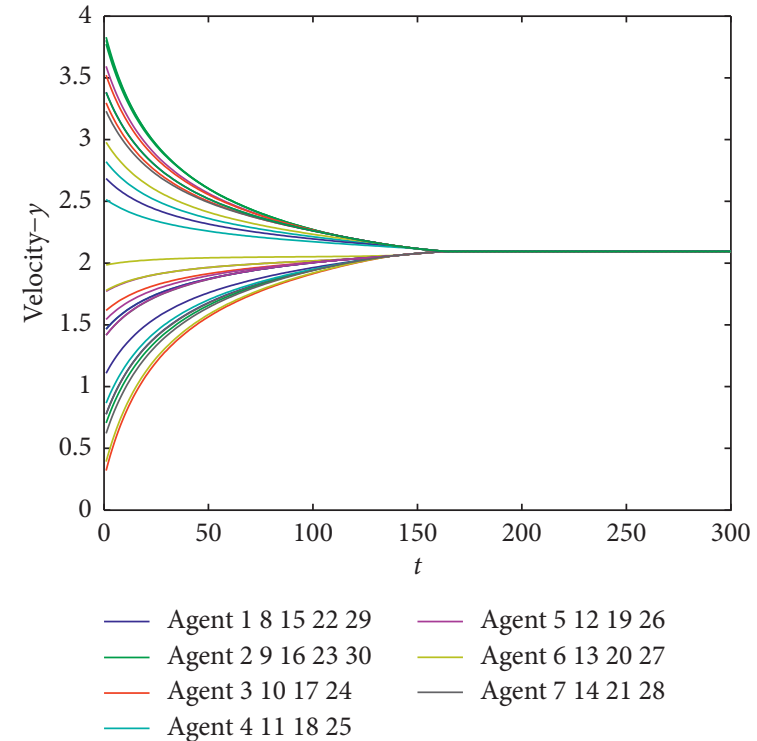

(b)

Figure 1: The velocity of agents.

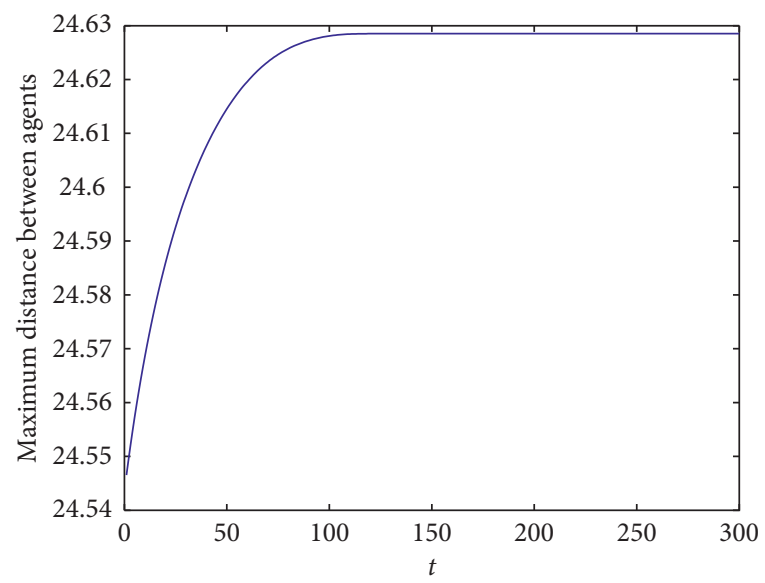

(a)

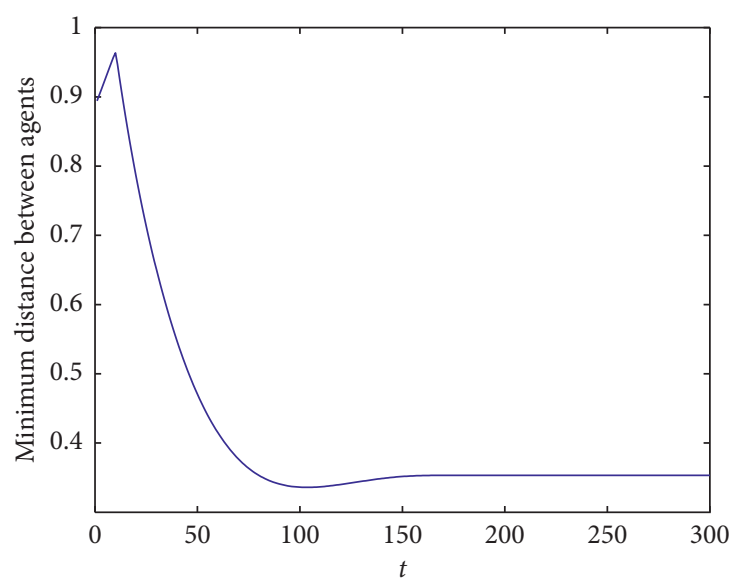

(b)

Figure 2: The maximum (a) and minimum (b) distance between agents.

where $\beta=0.2, r^{*}=3$, and $\psi^{*}=0.6310$. Using the formula in Theorem 1 , we see that $T^{*}=10.9824$ for the random initial position generated on $[0,20]$ and random velocity on $[0,4]$.

Then, the following simulation results (Figures 1 and 2) are obtained. In Figure 1, the $x, y$ direction velocity of the all agents is presented, and the velocity of all agents converges to the same value after about $T=1.5$. Moreover, in Figure 2, it shows that the maximum distance between all agents is stable after $T=1.5$. In the process of forming fixed-time flocking, the minimum distance among all agents is about 0.3359 for given random initial values.

\section{Conclusion}

In this paper, we investigated the flocking problem of a modified Cucker-Smale model with continuous non-Lipschitz protocol. By using a Lyapunov functional, we show that the flocking can occur in fixed time if communication rate function is locally Lipschitz continuous and with a lower bound. The main results demonstrate that the flocking converging time is independent of the initial states of agents. Theoretical results are supported by numerical simulations; at the same time, we observe that the minimum distance of the agent is only 0.339 , which is very dangerous. Therefore, 
avoiding the collision problem will guide significance to our further research studies.

\section{Data Availability}

The simulation data used to support the findings of this study are included within the article.

\section{Conflicts of Interest}

The authors declare that they have no conflicts of interest.

\section{Acknowledgments}

The authors would like to thank the reviewer for constructive comments. This work was supported by the National Natural Science Foundation of China (Grant no. 11671011).

\section{References}

[1] L. Pareschi and G. Toscani, Interacting Multiagent Systems: Kinetic Equations and Monte Carlo Methods, Oxford University Press, Oxford, UK, 2013.

[2] T. Vicsek and A. Zafeiris, "Collective motion," Physics Reports, vol. 517, no. 3-4, pp. 71-140, 2012.

[3] G. Albi and L. Pareschi, "Modeling of self-organized systems interacting with a few individuals: from microscopic to macroscopic dynamics," Applied Mathematics Letters, vol. 26, no. 4, pp. 397-401, 2013.

[4] M. R. D’Orsogna, Y. L. Chuang, A. L. Bertozzi, and L. S. Chayes, "Self-propelled particles with soft-core interactions: patterns, stability, and collapse," Physical Review Letters, vol. 96, no. 10, 2006.

[5] F. Cucker and S. Smale, "Emergent behavior in flocks," IEEE Transactions on Automatic Control, vol. 52, no. 5, pp. 852862, 2007.

[6] F. Cucker and S. Smale, "On the mathematics of emergence," Japanese Journal of Mathematics, vol. 2, no. 1, pp. 197-227, 2007.

[7] S.-Y. Ha and J.-G. Liu, "A simple proof of the Cucker-Smale flocking dynamics and mean-field limit," Communications in Mathematical Sciences, vol. 7, no. 2, pp. 297-325, 2009.

[8] S. Motsch and E. Tadmor, "A new model for self-organized dynamics and its flocking behavior," Journal of Statistical Physics, vol. 144, no. 5, pp. 923-947, 2011.

[9] Y. Liu and J. Wu, "Flocking and asymptotic velocity of the Cucker-Smale model with processing delay," Journal of Mathematical Analysis and Applications, vol. 415, no. 1, pp. 53-61, 2014.

[10] Y.-P. Choi and J. Haskovec, "Cucker-Smale model with normalized communication weights and time delay," Kinetic \& Related Models, vol. 10, no. 4, pp. 1011-1033, 2017.

[11] R. Erban, J. Haškovec, and Y. Sun, "A cucker--smale model with noise and delay," SIAM Journal on Applied Mathematics, vol. 76, no. 4, pp. 1535-1557, 2016.

[12] C. Pignotti and I. Reche Vallejo, "Flocking estimates for the Cucker-Smale model with time lag and hierarchical leadership," Journal of Mathematical Analysis and Applications, vol. 464, no. 2, pp. 1313-1332, 2018.

[13] F. Cucker and J.-G. Dong, "Avoiding collisions in flocks," IEEE Transactions on Automatic Control, vol. 55, no. 5, pp. 1238-1243, 2010.
[14] S. M. Ahn, H. Choi, S.-Y. Ha, and H. Lee, "On collisionavoiding initial configurations to Cucker-Smale type flocking models," Communications in Mathematical Sciences, vol. 10, no. 2, pp. 625-643, 2012.

[15] J. A. Carrillo, Y.-P. Choi, P. B. Mucha, and J. Peszek, "Sharp conditions to avoid collisions in singular Cucker-Smale interactions," Nonlinear Analysis: Real World Applications, vol. 37, pp. 317-328, 2017.

[16] H. Liu, X. Wang, Y. Liu, and X. Li, "On non-collision flocking and line-shaped spatial configuration for a modified singular Cucker-Smale model," Communications in Nonlinear Science and Numerical Simulation, vol. 75, pp. 280-301, 2019.

[17] J. Shen, "Cucker-Smale flocking under hierarchical leadership," SIAM Journal on Applied Mathematics, vol. 68, no. 3, pp. 694-719, 2008.

[18] F. Dalmao and E. Mordecki, "Cucker-Smale flocking under hierarchical leadership and random interactions," SIAM Journal on Applied Mathematics, vol. 71, no. 4, pp. 1307-1316, 2009.

[19] F. Dalmao and E. Mordecki, "Hierarchical cucker-smale model subject to random failure," IEEE Transactions on Automatic Control, vol. 57, no. 7, pp. 1789-1793, 2012.

[20] J.-G. Dong, "Flocking under hierarchical leadership with a free-will leader," International Journal of Robust \& Nonlinear Control, vol. 23, no. 16, pp. 1891-1898, 2013.

[21] Y. Han, D. Zhao, and Y. Sun, "Finite-time flocking problem of a Cucker-smale-type self-propelled particle model," Complexity, vol. 21, no. 1, pp. 354-361, 2015.

[22] H. Liu, X. Wang, X. Li, and Y. Liu, "Finite-time flocking and collision avoidance for second-order multi-agent systems," International Journal of Systems Science, vol. 51, no. 1, pp. 102-115, 2020.

[23] Z. Zuo, "Nonsingular fixed-time consensus tracking for second-order multi-agent networks," Automatica, vol. 54, pp. 305-309, 2015.

[24] B. Zhang and Y. Jia, "Fixed-time consensus protocols for multi-agent systems with linear and nonlinear state measurements," Nonlinear Dynamics, vol. 82, no. 4, pp. 16831690, 2015.

[25] B. Ning, Q.-L. Han, and Z. Zuo, "Distributed optimization for multiagent systems: an edge-based fixed-time consensus approach," IEEE Transactions on Cybernetics, vol. 49, no. 1, pp. 122-132, 2019.

[26] H. Hong, H. Wang, Z. Wang, and W. Yu, "Finite-time and fixed-time consensus problems for second-order multi-agent systems with reduced state information," Science China Information Sciences, vol. 62, no. 11, pp. 95-105, 2019.

[27] A. Polyakov, "Nonlinear feedback design for fixed-time stabilization of linear control systems," IEEE Transactions on Automatic Control, vol. 57, no. 8, pp. 2106-2110, 2012.

[28] G. H. Hardy, J. E. Littlewood, and G. Polya, Inequalities, Cambridge University Press, Cambridge, UK, 1952. 